The effect of surface tension on trapped modes in water-wave problems

Harter, R. and Abrahams, I. David and Simon, M. J. 2007

MIMS EPrint: 2007.212

Manchester Institute for Mathematical Sciences

School of Mathematics

The University of Manchester

\footnotetext{
Reports available from: http://eprints.maths.manchester.ac.uk/

And by contacting: The MIMS Secretary

School of Mathematics

The University of Manchester

Manchester, M13 9PL, UK
} 


\title{
The effect of surface tension on trapped modes in water-wave problems
}

\author{
By Robert Harter, I. David Abrahams* and Michael J. Simon \\ School of Mathematics, The University of Manchester, Oxford Road, \\ Manchester M13 9PL, UK
}

In this paper the effect of surface tension is considered on two two-dimensional waterwave problems involving pairs of immersed bodies. Both models, having fluid of infinite depth, support localized oscillations, or trapped modes, when capillary effects are excluded. The first pair of bodies is surface-piercing whereas the second pair is fully submerged. In the former case it is shown that the qualitative nature of the streamline shape is unaffected by the addition of surface tension in the free surface condition, no matter how large this parameter becomes. The main objective of this paper, however, is to study the submerged body problem. For this case it is found, by contrast, that there exists a critical value of the surface tension above which it is no longer possible to produce a completely submerged pair of bodies which support trapped modes. This critical value varies as a function of the separation of the two bodies. It can be inferred from this that surface tension does not always play a qualitatively irrelevant role in the linear water-wave problem.

Keywords: water waves; trapped modes; surface tension; submerged body; localized oscillations

\section{Introduction}

For over half a century, there has been considerable interest in answering the question of under what conditions the two-dimensional linear water-wave problem (with a prescribed inhomogeneous boundary condition) admits a unique mathematical solution. As is usual, a uniqueness proof aims to show that the difference problem in which the boundary conditions are homogeneous (i.e. the problem for the difference between two solutions of the previous problem) has only a trivial solution. Although a general uniqueness proof is now known to be unobtainable, there have been many partial results proving the uniqueness of certain configurations or classes of systems. For example, Ursell (1950) has proved uniqueness in the case of a submerged horizontal cylinder lying in infinitely deep fluid, immersed to any depth, for all frequencies. More recently, Simon \& Ursell (1984) have provided a uniqueness proof for any two-dimensional system of submerged obstacles that are contained by lines, emanating from some point on the free surface, that make $45^{\circ}$ angles with the horizontal. Until recently, it was generally believed that the uniqueness proof for all configurations * Author for correspondence (i.d.abrahams@manchester.ac.uk). 
would be possible to construct; however, this view was dispelled by McIver (1996), who provided the first example of non-uniqueness. In that paper, the so-called 'inverse method' was used to construct a difference velocity potential that satisfies the linear water-wave problem. This potential arises from a pair of sources placed symmetrically on the free surface, and the sources are chosen to be separated by a half wavelength so that the potential does not radiate waves to infinity. Furthermore, there are symmetric pairs of streamlines that separate the sources from the fluid, any of which can be interpreted as the boundaries of surface-piercing bodies. The potential represents a non-trivial solution of the linearized water-wave problem. Since the problem is linear, any scalar multiple of this solution is also a solution, thus proving non-uniqueness. This was the first example of a 'trapped-mode' solution in two-dimensional free surface water-wave problems, so-called because the energy of the motion is confined around the bodies.

More recently, a greater emphasis has been placed on finding other explicit examples of trapped modes. The first example of trapped modes involving a submerged obstacle was provided by Evans \& Porter (1998). However, this configuration also included surface-piercing bodies. The first example of a trapped mode involving only submerged bodies was given by McIver (2000), who used the same inverse procedure previously outlined. This time, rather than using a pair of sources, a symmetric combination of submerged dipoles was placed in the flow, with strengths chosen to guarantee wave cancellation at infinity. Examples of three-dimensional structures that support trapped modes are also known. A common feature of these bodies is that they isolate a portion of the free surface. The three-dimensional analogue of McIver's (1996) solution was found by McIver \& McIver (1997). In that paper it is shown that a family of surface-piercing tori support localized oscillations. More recently, McIver \& Porter (2002) have provided numerical evidence to suggest that a submerged torus can support trapped modes, and McIver \& Newman (2003) have found examples of non-axisymmetric structures that admit non-unique solutions.

None of the results documented above accounts for the effect of surface tension. Indeed, to the authors' knowledge, no previous investigation has been made on the existence of trapped modes in an unbounded fluid layer in the presence of surface tension. For simplicity, the focus of this paper will be on the twodimensional surface-piercing and submerged examples examined by McIver. The results will be recreated with surface tension added to see what effect, if any, this will have. The aim of this is to enhance understanding of whether it is physically realistic to exclude surface tension from the linear water-wave problem. For both of these problems, a two-dimensional Cartesian coordinate system $(x, y)$ is adopted, with the $y$-axis pointing vertically downwards. The fluid is inviscid and its depth is considered infinite. In the section to follow, for trapped-mode solutions, a time-harmonic velocity potential of the form $\operatorname{Re}\left\{\phi(x, y)^{-i \omega t}\right\}$ is sought, where $\omega$ is the angular frequency; $K=\omega^{2} / g$ is the wavenumber for free surface waves in the absence of surface tension; $\rho$ is the fluid density; and $g$ is the acceleration due to gravity. For a trapped mode to exist, the difference velocity potential $\phi(x, y)$ must satisfy the homogeneous boundary value problem

$$
\nabla^{2} \phi=0 \text { in the fluid, }
$$




$$
\begin{gathered}
\frac{\partial \phi}{\partial y}+K \phi-\frac{T}{\rho g} \frac{\partial^{3} \phi}{\partial x^{2} \partial y}=0 \quad \text { on the free surface, } \\
\frac{\partial \phi}{\partial n}=0 \quad \text { on fixed, rigid boundaries and } \\
|\nabla \phi| \rightarrow 0 \quad \text { as } x^{2}+y^{2} \rightarrow \infty(y \geq 0) .
\end{gathered}
$$

The flow is considered to be incompressible and irrotational, which results in equation (1.1). Equation (1.2) is the linearized free surface condition including surface tension $T$ (Billingham \& King 2000). Equation (1.3) states that there is no flow through stationary impenetrable boundaries, and condition (1.4) stipulates that no waves are radiated to infinity.

Equations (1.1) and (1.2) allow propagating wave solutions of the form $\exp \left( \pm \mathrm{i} k_{0} x-k_{0} y\right)$, where the wavenumber $k_{0}$ is the positive real root of

$$
\frac{T k_{0}^{3}}{\rho g}+k_{0}-K=0 \text {. }
$$

It is convenient to non-dimensionalize the system (1.1)-(1.5) using $1 / k_{0}$ as the length-scale, thereby yielding a modified free surface condition

$$
\frac{\partial \phi}{\partial y}+(1+s) \phi-s \frac{\partial^{3} \phi}{\partial x^{2} \partial y}=0
$$

where $s=T k_{0}^{2} / \rho g=\left(K / k_{0}\right)-1$ is a measure of the surface tension for fixed $\omega, \rho$ and $g$. Equations (1.1), (1.2) and (1.4) are left unchanged by this transformation of $k_{0}(x, y) \rightarrow(x, y)$.

The outline of the paper is as follows: in $§ 2$ the work by McIver (1996) on surface-piercing bodies is extended to include surface tension, and it is shown that the topological nature of the streamlines is unchanged. For simplicity, satisfaction of a contact-line condition is ignored in this section; the work is presented primarily for ease of exposition of the solution procedure. However, as will be shown in a forthcoming article by Harter et al. (in preparation), the results are still valid (away from the contact points) for small values of surface tension $s$. In $\S 3$ the submerged bodies presented in McIver (2000) are considered, and corresponding results are given for $s \neq 0$. It is noted that the initially highly accurate numerical results break down in a particular s-region, and in $\S 4$ asymptotic expansions are used to confirm that this is because there is a critical value of $s$ above which these trapped modes can no longer be constructed. In $\S 5$ we investigate how the solution behaves when other parameters are allowed to vary, with some concluding remarks in $\$ 6$.

\section{Trapped modes around surface-piercing bodies}

To derive a potential equivalent to that given by McIver (1996), the method of Thorne (1953) is followed closely with the straightforward addition of surface tension. A two-dimensional velocity potential due to a source (i.e. a logarithmic 
singularity) positioned at $(0, f)$, where $f \geq 0$, is sought in the form

$$
\Phi(x, y, t ; s)=\left(\frac{1}{2} \ln \frac{r}{r^{\prime}}+\phi_{1}(x, y)\right) \cos \omega t+\phi_{2}(x, y, t),
$$

where $r^{2}=x^{2}+(y-f)^{2}$ and $r^{\prime 2}=x^{2}+(y+f)^{2}$. The harmonic functions $\phi_{1}$ and $\phi_{2}$ are such that both $\left((1 / 2) \ln \left(r / r^{\prime}\right)+\phi_{1}\right)$ and $\phi_{2}$ satisfy equation (1.6), and $\phi_{2}$ is chosen so that $\Phi$ represents an outgoing wave-train at infinity. It turns out that

$$
\begin{aligned}
\Phi(x, y, t ; s)= & \left(\frac{1}{2} \ln \frac{r}{r^{\prime}}+f_{0}^{\infty} \frac{1+s m^{2}}{1+s-m-s m^{3}} \mathrm{e}^{-m(y+f)} \cos m x \mathrm{~d} m\right) \cos \omega t \\
& -\pi \frac{1+s}{3 s+1} \mathrm{e}^{-(y+f)} \cos x \sin \omega t .
\end{aligned}
$$

Here, the integral has a singularity at $m=1$, and so is to be interpreted as a Cauchy principal-value. In particular, when $f=0$, a time-harmonic source potential of the form $\operatorname{Re}\left\{\phi(x, y ; s) \mathrm{e}^{-\mathrm{i} \omega t}\right\}$ can be considered, where

$$
\phi(x, y ; s)=\phi_{1}+\phi_{2}=\oint_{0}^{\infty} \frac{1+s m^{2}}{s m^{3}+m-1-s} \mathrm{e}^{-m y} \cos m x \mathrm{~d} m,
$$

in which the integral sign $f$ here and henceforth indicates deformation of the contour below the pole. Note that the principal-value component of this expression is $\phi_{1}$ and the semicircular loop around the singularity at $m=1$ yields $\phi_{2}$. Expression (2.3) can be split into three via partial fractions as

$$
\left.\phi(x, y ; s)=\frac{1}{3 s+1}\right\}_{0}^{\infty}\left\{\frac{s+1}{m-1}+\frac{A_{+}}{m-m_{+}}+\frac{A_{-}}{m-m_{-}}\right\} \mathrm{e}^{-m y} \cos m x \mathrm{~d} m,
$$

where

$$
m_{ \pm}=-\frac{1}{2} \pm \frac{\mathrm{i} v}{2} \quad \text { and } \quad A_{ \pm}=s \pm \frac{\mathrm{i}}{v} \quad \text { with } \quad v=\sqrt{3+\frac{4}{s}} .
$$

Clearly, each of the three resulting integrals has an integrand of the form $\alpha \exp (-m y) \cos m x /\left(m-m_{0}\right)$, where for the first the constants $\alpha$ and $m_{0}$ are real but for the latter two they are complex. The integrals can be evaluated easily using the identities

$$
f_{0}^{\infty} \frac{\mathrm{e}^{\mathrm{i} m z}}{m-m_{0}} \mathrm{~d} m=\mathrm{e}^{\mathrm{i} m_{0} z} \begin{cases}\mathrm{E}_{1}\left(\mathrm{i} m_{0} z\right)-\pi \mathrm{i} & \left(m_{0} \in \mathbb{R}^{+}, \operatorname{Re} z<0\right), \\ \mathrm{E}_{1}\left(\mathrm{i} m_{0} z\right)+\pi \mathrm{i} & \left(m_{0} \in \mathbb{R}^{+}, \operatorname{Re} z>0\right), \\ \mathrm{E}_{1}\left(\mathrm{i} m_{0} z\right) & \left(m_{0} \notin \mathbb{R}^{+}\right) .\end{cases}
$$

Note that the principal-value sign is only required for the case $m_{0} \in \mathbb{R}^{+}$, and that $E_{1}$ is the exponential integral, defined by

$$
E_{1}(z)=\int_{z}^{\infty} \frac{\mathrm{e}^{-t}}{t} \mathrm{~d} t, \quad|\arg z|<\pi,
$$

which has a branch cut along the negative real axis. Finally, it is straightforward to show by contour integration that, as $|x| \rightarrow \infty$,

$$
\phi(x, y ; s) \sim \mathrm{i} \pi \frac{s+1}{3 s+1} \mathrm{e}^{-y+\mathrm{i}|x|} .
$$


Now that the form of the source potential $\phi$ and its wave radiation are known, McIver's streamline plots can be recreated, and equivalent figures produced when capillary effects are included.

It is worth reiterating at this stage that the inclusion of surface tension in the free surface condition alone is insufficient, as there must be a condition at the line of contact between the free surface and any surface-piercing body. Unfortunately, the contact-line problem is not well understood by the academic community, especially for oscillatory motion, and no agreement has been reached on exactly what condition should be applied there (for several studies on this point, the reader is referred to articles by Evans (1968), Hocking (1987) and Rhodes-Robinson (1991)). For the above reasons, the present study will not include an extra condition to be satisfied at the contact line; implicitly, this results in satisfaction of the condition of continuity of surface elevation and slope at the contact point (between fluid in the physical and image regions). The current authors have recently completed work which takes the present surface-piercing model and includes one possible contact-line condition (Harter et al. in preparation), which is the case where the free surface is 'pinned' to the surface-piercing bodies.

In the present paper the work of McIver (1996) is considered first because it allows us to show how surface tension can be included in the source potential and stream function, and how these expressions can be dealt with analytically and evaluated exactly. Although the solution here for general $s$ may not be entirely realistic, it is also reasonable to suppose that for weak surface tension the contact-line condition may have little effect, as is borne out by the authors' previously mentioned forthcoming article (Harter et al. in preparation). The main emphasis here is on an extension of the work of McIver (2000) for which the bodies are submerged and therefore there is no contact line involved.

McIver (1996) considers two equal sources at $( \pm a, 0)$ which produce the nondimensionalized velocity potential

$$
\phi_{0}(x, y)=\phi(x+a, y ; 0)+\phi(x-a, y ; 0) ;
$$

here, the subscript zero denotes the absence of surface tension (where $s$ and hence $A_{ \pm}$vanish). Clearly, as $|x| \rightarrow \infty$,

$$
\phi_{0}(x, y) \sim \pi \mathrm{i} \mathrm{e}^{\mathrm{i}|x+a|-y}+\pi \mathrm{i} \mathrm{e}^{\mathrm{i}|x-a|-y}=2 \pi \mathrm{i} \mathrm{e}^{\mathrm{i}|x|-y} \cos a
$$

and so the choice $a=\pi / 2$ ensures that no waves are radiated to infinity. This therefore satisfies (1.1), (1.4) and (1.6) (with $s=0)$. The corresponding stream function is given by

$$
\psi_{0}(x, y)=\oint_{0}^{\infty} \frac{\mathrm{e}^{-k y}}{k-1} \sin k(x+a) \mathrm{d} k+\int_{0}^{\infty} \frac{\mathrm{e}^{-k y}}{k-1} \sin k(x-a) \mathrm{d} k
$$

and typical streamlines of this flow are shown in figure 1 for $a=\pi / 2$. It can be seen that there are pairs of streamlines, emanating from the sources, which are inclined above the free surface and so separate these singular points from the fluid. These streamlines can be interpreted as the boundaries of two surfacepiercing bodies that support trapped modes.

When $s \neq 0$ a wave-free potential can be constructed in an identical way, namely by placing equal sources at $( \pm \pi / 2,0)$ to give (ignoring a multiplicative 


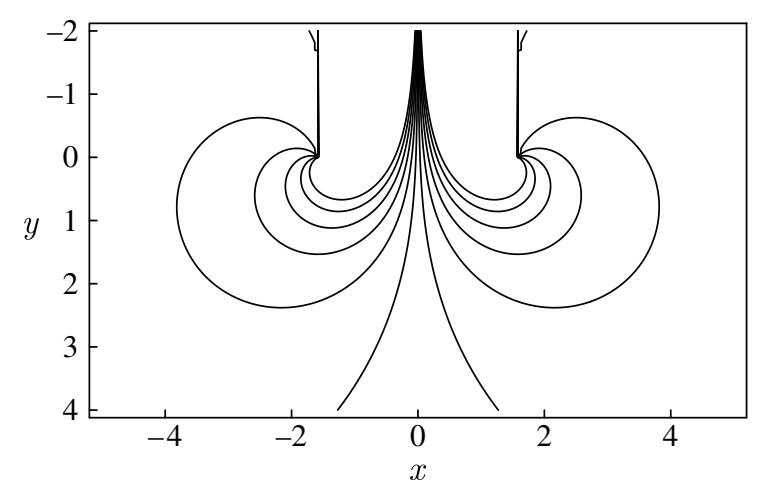

Figure 1. Recreation of the streamlines originally produced by McIver (1996).

constant) a stream function

$$
\begin{aligned}
\psi_{s}(x, y) & =\psi_{0}^{\infty}\left\{\frac{s+1}{m-1}+\frac{A_{+}}{m-m_{+}}+\frac{A_{-}}{m-m_{-}}\right\} \mathrm{e}^{-m y}\left(\sin m\left(x+\frac{\pi}{2}\right)+\sin m\left(x-\frac{\pi}{2}\right)\right) \mathrm{d} m \\
& =2 \psi_{0}^{\infty}\left\{\frac{s+1}{m-1}+\frac{A_{+}}{m-m_{+}}+\frac{A_{-}}{m-m_{-}}\right\} \mathrm{e}^{-m y} \sin (m x) \cos (m \pi / 2) \mathrm{d} m ;
\end{aligned}
$$

here, the pole at $m=1$ has been cancelled out, and the constants $m_{ \pm}$and $A_{ \pm}$are as in (2.4). This expression for $\psi_{s}(x, y)$ can again be evaluated via $(2.5)$. The streamline patterns for a few values of $s$ are shown in figure 2 .

It can be seen that the inclusion of surface tension does not change the streamline patterns greatly, and this has been verified for large values of $s$. In fact, it is known (Lighthill 1978, p. 225) that surface tension is the 'dominant' effect in water waves of wavelength smaller than approximately $4 \mathrm{~mm}$, corresponding to $k_{0} \gtrsim 1600 \mathrm{~m}^{-1}$, and so $s=T k_{0}^{2} / \rho g \gtrsim 18.6$ (for water, in which $T \approx 0.074 \mathrm{~N} \mathrm{~m}^{-1}$ and $\rho \approx 1000 \mathrm{~kg} \mathrm{~m}^{-3}$, along with $\left.g \approx 9.81 \mathrm{~m} \mathrm{~s}^{-2}\right)$. Whatever the effects of surface tension are, it may be expected that they would be seen for $s$ at approximately 20 . However, none of the graphs in figure 2 shows a dramatically different nature - there are still two surface-piercing bodies that enclose the source points.

\section{Trapped modes around submerged bodies}

For the submerged case, McIver (2000) constructs a velocity potential, consisting of pairs of vertical and horizontal dipoles, which satisfies (1.1), (1.4) and (1.6) (with $s=0)$. The dipoles are positioned symmetrically at $( \pm \pi / 4, h)$, where $h$ is allowed to vary. It is observed that, for each $h>0$, the flow has four saddle-point stagnation points that lie in the fluid - two in $x>0$ and two in $x<0$. Considering the region $x>0$, it is argued that there exists a positive value of $h$, say $h_{0}$, that will ensure that the stream function takes the same value, say $\psi_{0}$, at the two stagnation points when the dipoles are positioned at $\left(\pi / 4, h_{0}\right)$. This means that a streamline connects the two stagnation points and, it is shown, forms a closed loop. The argument involves finding positive numbers $h_{1}<h_{0}$ and $h_{2}>h_{0}$ such that 
(a)

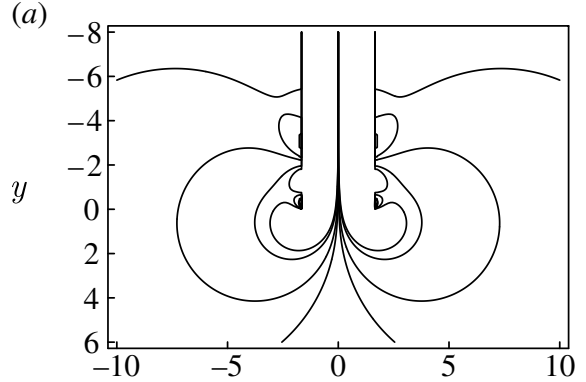

(c)

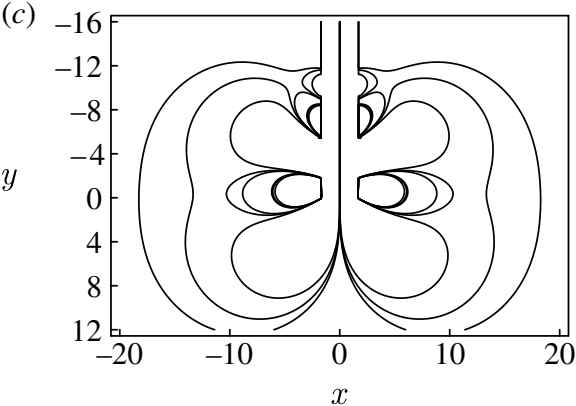

(b)

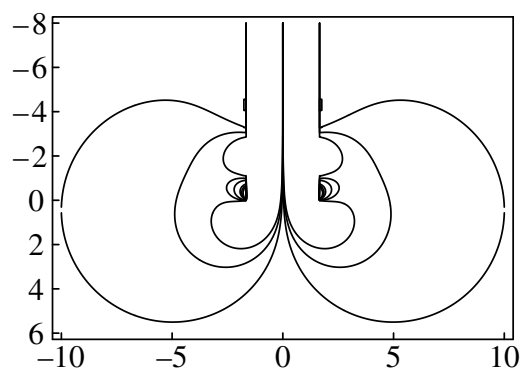

$(d)$

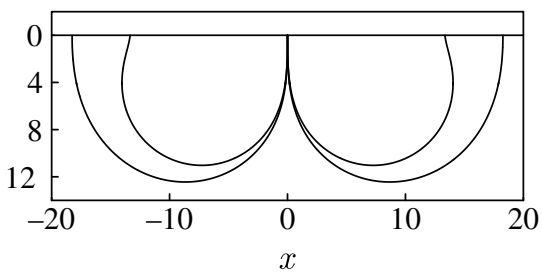

Figure 2. The effect of surface tension on McIver's streamlines when $(a) s=0.4,(b) s=1$ and (c) $s=20 .(d)$ For this last value of surface tension, typical streamlines that can be interpreted as surface-piercing bodies are shown.

$\psi_{1}-\psi_{2}<0$ for $h=h_{1}$ and $\psi_{1}-\psi_{2}>0$ for $h=h_{2}$ (where $\psi_{1}$ and $\psi_{2}$ are the respective stream function values at the stagnation points). Since $\psi$ is a continuously varying function of $h$, there must be a value of $h$ between $h_{1}$ and $h_{2}$ where $\psi_{1}-\psi_{2}=0$. An equivalent argument shows that the dipoles in $x<0$ need to be positioned at $\left(-\pi / 4, h_{0}\right)$. The constant $h_{0}$ is found numerically to be approximately equal to 0.0547846 . When the dipoles are positioned at $\left( \pm \pi / 4, h_{0}\right)$, they are enclosed by a pair of streamlines. These streamlines may therefore be interpreted as a pair of submerged bodies. To prove that they are submerged, a plot of $\psi$ along the free surface shows that $\psi(x, 0)=\psi_{0}$ for two values of $x$. These values correspond to the other branch of the saddle not being interpreted as a body contour (figure 3).

It can also be shown that the stagnation points lie in the fluid by invoking the argument principle. Let $w(z)=\phi+\mathrm{i} \psi$ be the complex potential and $\Gamma$ be a positively orientated closed curve. If it is assumed that $w^{\prime}(z)$ is meromorphic inside $\Gamma$, and analytic and non-zero on $\Gamma$, then

$$
\frac{1}{2 \pi \mathrm{i}} \oint_{\Gamma} \frac{w^{\prime \prime}(z)}{w^{\prime}(z)} \mathrm{d} z=\sum_{i=1}^{m} z_{i}-\sum_{j=1}^{n} p_{j},
$$

where $z_{i}$ and $p_{j}$ are the multiplicities of the $m$ zeros and the orders of the $n$ poles that lie inside $\Gamma$, respectively. By choosing $\Gamma$ to be the $x$ and $y$ axes with a quarter circle of radius $R$, the existence of stagnation points in the first quadrant can be verified by noting that there are two values of $R$ for which a small increase in $R$ leads to a jump of +1 in the value of the integral. The number and magnitude of these jumps arise due to the fact that $w^{\prime}(z)$ has two simple zeros in this region. 


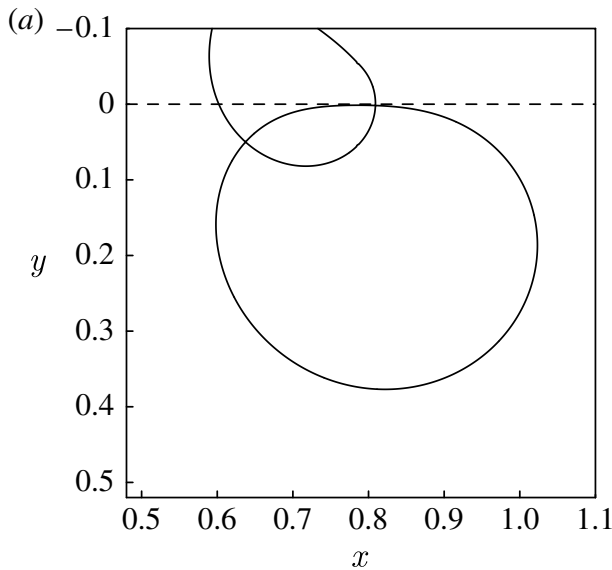

(b)

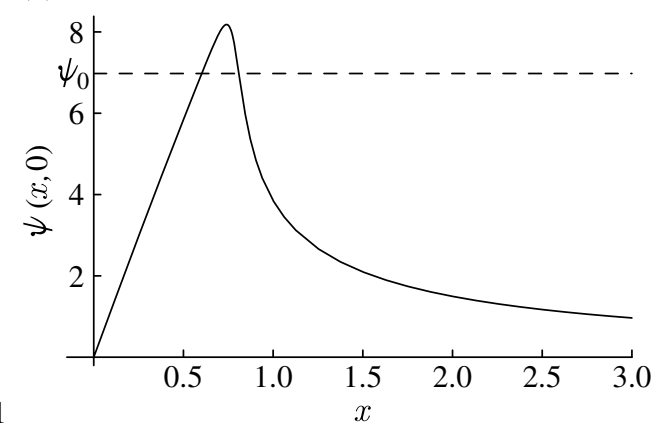

Figure 3. (a) The submerged body in $x>0$ that supports trapped modes and (b) a plot of the stream function values on the free surface, both presented in McIver (2000).

It may be queried that, for submerged obstacles lying so close to the free surface (figure 3), viscous as well as surface tension effects would be important. If this were the case then localized oscillations would soon be dissipated. However, coordinates are scaled on the wavenumber $k_{0}(1.5)$, and so for long surface waves (low frequency) the submergence depth need not be small but could be of order unity or larger. It thus seems reasonable that viscous effects can be neglected when generalizing McIver's (2000) study and attention is now turned to the case when surface tension only is included; expressions for the potentials for horizontal and vertical dipoles, positioned at $(\xi, \eta)$, are now required. The reader may verify by direct substitution into (1.1) and (1.6) that these are given respectively by

$$
\begin{aligned}
(1+s) \phi_{h}(x, y, \xi, \eta)= & \frac{x-\xi}{(x-\xi)^{2}+(y-\eta)^{2}}+\frac{x-\xi}{(x-\xi)^{2}+(y+\eta)^{2}} \\
& +2(1+s) \int_{0}^{\infty} \frac{\mathrm{e}^{-m(y+\eta)} \sin m(x-\xi)}{s m^{3}+m-1-s} \mathrm{~d} m
\end{aligned}
$$

and

$$
\begin{aligned}
(1+s) \phi_{v}(x, y, \xi, \eta)= & \frac{y-\eta}{(x-\xi)^{2}+(y-\eta)^{2}}-\frac{y+\eta}{(x-\xi)^{2}+(y+\eta)^{2}} \\
& -2(1+s) \psi_{0}^{\infty} \frac{\mathrm{e}^{-m(y+\eta)} \cos m(x-\xi)}{s m^{3}+m-1-s} \mathrm{~d} m .
\end{aligned}
$$

These velocity potentials may be derived by Thorne's (1953) method or by direct differentiation of $(2.2)$ in the case $f \neq 0$. The corresponding stream functions are

$$
\begin{aligned}
(1+s) \psi_{h}(x, y, \xi, \eta)= & -\frac{y-\eta}{(x-\xi)^{2}+(y-\eta)^{2}}-\frac{y+\eta}{(x-\xi)^{2}+(y+\eta)^{2}} \\
& -2(1+s)\}_{0}^{\infty} \frac{\mathrm{e}^{-m(y+\eta)} \cos m(x-\xi)}{s m^{3}+m-1-s} \mathrm{~d} m
\end{aligned}
$$


and

$$
\begin{aligned}
(1+s) \psi_{v}(x, y, \xi, \eta)= & \frac{x-\xi}{(x-\xi)^{2}+(y-\eta)^{2}}-\frac{x-\xi}{(x-\xi)^{2}+(y+\eta)^{2}} \\
& -2(1+s) \psi_{0}^{\infty} \frac{\mathrm{e}^{-m(y+\eta)} \sin m(x-\xi)}{s m^{3}+m-1-s} \mathrm{~d} m .
\end{aligned}
$$

It can be shown that as $|x-\xi| \rightarrow \infty$,

$$
\left\{\begin{array}{l}
\phi_{h} \\
\phi_{v}
\end{array}\right\} \rightarrow \frac{2 \pi}{3 s+1} \mathrm{e}^{-(y+\eta)}\left\{\begin{array}{c}
\operatorname{sgn}(x-\xi) \mathrm{e}^{\mathrm{i}|x-\xi|} \\
-\mathrm{i} \mathrm{e}^{\mathrm{i}|x-\xi|}
\end{array}\right\} .
$$

Thus, it follows that the combination

$$
\begin{aligned}
\phi(x, y, a, h)= & \sin a\left\{\phi_{v}(x, y, a, h)+\phi_{v}(x, y,-a, h)\right\} \\
& -\cos a\left\{\phi_{h}(x, y, a, h)-\phi_{h}(x, y,-a, h)\right\}
\end{aligned}
$$

does not radiate waves to infinity. This potential represents a horizontal and vertical dipole combination positioned at $(a, h)$, together with an identical pairing at $(-a, h)$. The stream function for this oscillatory flow is given by

$$
\begin{aligned}
\psi(x, y, a, h)= & \sin a\left\{\psi_{v}(x, y, a, h)+\psi_{v}(x, y,-a, h)\right\} \\
& -\cos a\left\{\psi_{h}(x, y, a, h)-\psi_{h}(x, y,-a, h)\right\} .
\end{aligned}
$$

McIver's result is obtained by setting $s=0$ in the above. It should also be noted that both $a$ and $h$ are arbitrary. McIver chose $a=\pi / 4$ because the submerged bodies that support trapped modes are most deeply submerged when $a$ is near this value. Later the case where $a$ is allowed to vary will be considered.

It is convenient to work with the complex potential $w(z)=\phi+\mathrm{i} \psi$, which is given by

$$
\begin{aligned}
w(z)= & \frac{-2 z_{0} \mathrm{e}^{-\mathrm{i} a}}{(1+s)\left(z^{2}-z_{0}^{2}\right)}-\frac{2 \bar{z}_{0} \mathrm{e}^{\mathrm{i} a}}{(1+s)\left(z^{2}-\bar{z}_{0}^{2}\right)}-2 \mathrm{ie}^{-\mathrm{i} a} \int_{0}^{\infty} \frac{\mathrm{e}^{\mathrm{i} m\left(z+z_{0}\right)}}{s m^{3}+m-1-s} \mathrm{~d} m \\
& +2 \mathrm{ie} \mathrm{i}^{\mathrm{i} a} \int_{0}^{\infty} \frac{\mathrm{e}^{\mathrm{i} m\left(z-\bar{z}_{0}\right)}}{s m^{3}+m-1-s} \mathrm{~d} m,
\end{aligned}
$$

where $z=x+\mathrm{i} y, z_{0}=a+\mathrm{i} h$ and $\bar{z}_{0}$ is the complex conjugate of $z_{0}$. The two integrals in this expression can, as in $\$ 2$, be decomposed via the use of partial fractions

$$
\frac{1}{s m^{3}+m-1-s}=\frac{1}{3 s+1}\left(\frac{1}{m-1}+\frac{B_{+}}{m-m_{+}}+\frac{B_{-}}{m-m_{-}}\right)
$$

where

$$
B_{ \pm}=-\frac{1}{2} \pm \frac{3 \mathrm{i}}{2 v} \quad\left(\text { with } \quad m_{ \pm}=-\frac{1}{2} \pm \frac{\mathrm{i} v}{2} \quad \text { and } \quad v=\sqrt{3+\frac{4}{s}} \text { as before }\right) .
$$

Thus, the complex potential in (3.7) requires the evaluation of integrals of the form given in (2.5). As before, either $m_{0}=1$ or $2 m_{0}=-1 \pm \mathrm{i} v$. Define

$$
g(z)=\int_{0}^{\infty} \frac{\mathrm{e}^{\mathrm{i} m z}}{m-1} \mathrm{~d} m=\mathrm{e}^{\mathrm{i} z}\left(\mathrm{E}_{1}(\mathrm{i} z) \pm \pi \mathrm{i}\right), \quad \pm \operatorname{Re}(z)>0 .
$$


From the definition of $E_{1}(z)$ and integrals of the form (2.5), it is clear that the complex potential when evaluated will have branch cuts where $\arg \left(\mathrm{i} m_{ \pm}\left(z+z_{0}\right)\right)=\pi$ and $\arg \left(i m_{ \pm}\left(z-\bar{z}_{0}\right)\right)=\pi$, i.e. the two branch points lie in $y<0$ outside the fluid domain. It is therefore convenient to define alternative branches $p_{ \pm}(z)$ of the integral (2.5) such that the branch cut of each function lies vertically upwards. Specifically, this is achieved by writing

$$
\begin{aligned}
& p_{+}(z)=\mathrm{e}^{\mathrm{i} m_{+} z}\left\{\begin{array}{cc}
E_{1}\left(\mathrm{i} m_{+} z\right)+2 \pi \mathrm{i} & \left(-\frac{\pi}{2}<\arg (z)<-\arg \left(-\mathrm{i} m_{+}\right)\right), \\
E_{1}\left(\mathrm{i} m_{+} z\right) & (\text { otherwise }),
\end{array}\right. \\
& p_{-}(z)=\mathrm{e}^{\mathrm{i} m_{-} z}\left\{\begin{array}{cc}
E_{1}\left(\mathrm{i} m_{-} z\right)-2 \pi \mathrm{i} & \left(-\frac{\pi}{2}<\arg (-\bar{z})<\arg \left(\mathrm{i} m_{-}\right)\right), \\
E_{1}\left(\mathrm{i} m_{-} z\right) & \text { (otherwise) } .
\end{array}\right.
\end{aligned}
$$

It follows that the non-dimensionalized complex potential can be rewritten as

$$
\begin{aligned}
w(z)= & -\frac{2 z_{0} \mathrm{e}^{-\mathrm{i} a}}{(1+s)\left(z^{2}-z_{0}^{2}\right)}-\frac{2 \mathrm{ie}^{-\mathrm{i} a}}{3 s+1}\left(g\left(z+z_{0}\right)+B_{+} p_{+}\left(z+z_{0}\right)+B_{-} p_{-}\left(z+z_{0}\right)\right) \\
& -\frac{2 \bar{z}_{0} \mathrm{e}^{\mathrm{i} a}}{(1+s)\left(z^{2}-\bar{z}_{0}^{2}\right)}+\frac{2 \mathrm{ie}^{\mathrm{i} a}}{3 s+1}\left(g\left(z-\bar{z}_{0}\right)+B_{+} p_{+}\left(z-\bar{z}_{0}\right)+B_{-} p_{-}\left(z-\bar{z}_{0}\right)\right) .
\end{aligned}
$$

This form of the complex potential can be input into a mathematical software package, such as Mathematica, and the streamlines found by plotting the contours of its imaginary part. Of course, if $s=0$ then there are no complex zeros of $s m^{3}+m-1-s$, and it is not necessary to introduce the functions $p_{ \pm}(z)$. In this case, the complex potential reduces to

$$
w_{0}(z)=\frac{-2 z_{0} \mathrm{e}^{-\mathrm{i} a}}{\left(z^{2}-z_{0}^{2}\right)}-\frac{2 \bar{z}_{0} \mathrm{e}^{\mathrm{i} a}}{\left(z^{2}-\bar{z}_{0}^{2}\right)}-2 \mathrm{ie}^{-\mathrm{i} a} g\left(z+z_{0}\right)+2 \mathrm{e}^{\mathrm{i} a} g\left(z-\bar{z}_{0}\right) .
$$

Several results of this analysis are shown in figures 4-6 (for the remainder of this section, the case $a=\pi / 4$ will be considered). Figure 4 clearly shows that, as the value of $s$ is increased, the two stagnation points converge upon either side of $x=\pi / 4$ and move closer to the free surface. Figure 5 shows that the dipole submergence also becomes very small. The streamline plots for two values of $s$ are shown in figure 6 . It should be recalled that an equivalent body, lying in $x<0$, is also required for trapped modes to exist. Comparison with the streamline plot when $s=0$ (figure 3 ) shows that the branch of the saddle that crosses the free surface is gradually decreasing in size; indeed, when $s=1.1$ it cannot be seen on the contour plot, although magnified plots verify its continued existence. This again suggests that the stagnation points are moving closer together. It should also be noted that both the width and height of the submerged bodies increase with $s$. However, the bodies are very close to the free surface (when $s=1$, for example, both stagnation points are within $10^{-9}$ of the $x$-axis) and so they might be considered to be hardly submerged at all. Of course, the results given in McIver (2000) when $s=0$ were already very close to the free surface, but the inclusion of surface tension pushes them even closer. 

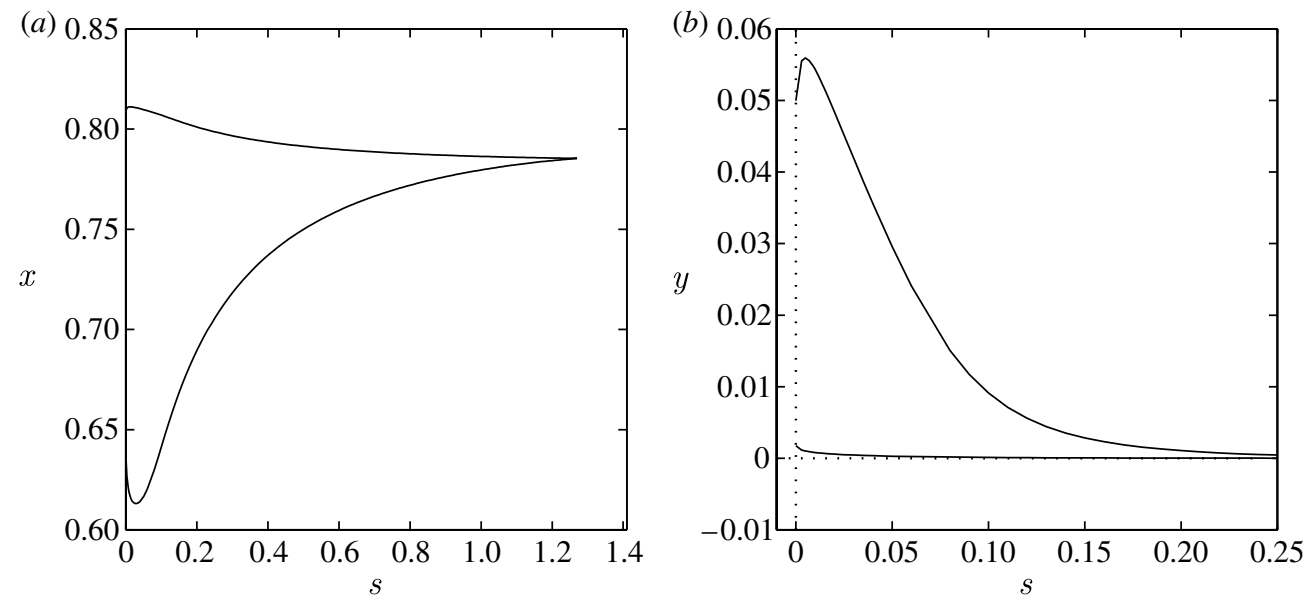

Figure 4. The $(a) x$ and $(b) y$ location of the stagnation points as a function of $s(a=\pi / 4)$.

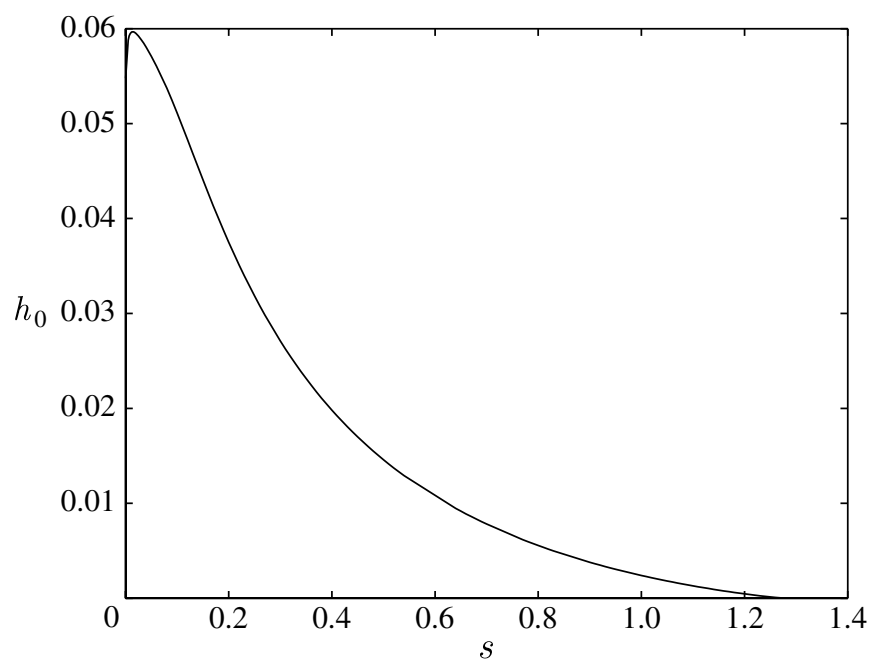

Figure 5. Dipole submergence that ensures $\psi_{1}=\psi_{2}$, as a function of $s$.

Another point concerning the numerical results is that, as $s$ is increased, the accuracy of the computed values of the stagnation points gradually decreases. This can be verified by checking the values of $\partial \phi / \partial x$ and $\partial \phi / \partial y$ at these points. However, this does not become a major problem until $s$ exceeds a value of approximately 1.27 , at which point the (previously small) values of $\partial \phi / \partial x$ and $\partial \phi / \partial y$ start to increase rapidly. Indeed, by around $s=1.275$ the results can no longer be taken to be accurate. This is not due to a problem with the numerical method being employed (Newton's method), rather it suggests that the nature of the solution changes close to this value of surface tension $s$, and this will be confirmed in $\$ 4$.

Finally, it should be noted that it is not possible to invoke the argument principle and to consider a free surface plot for all values of $s$ (and later for all values of $a$ ), in order to verify that the bodies are submerged. However, numerous checks have been made by the authors for particular values of $s$ and $a$ to convince themselves of the validity of the results. 
(a)

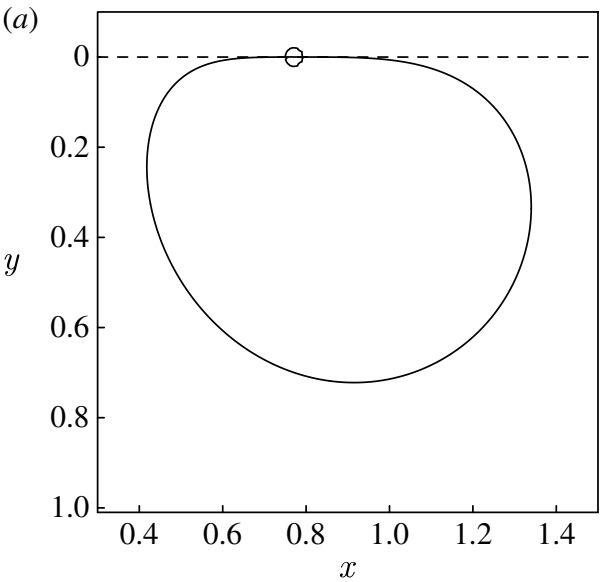

(b)

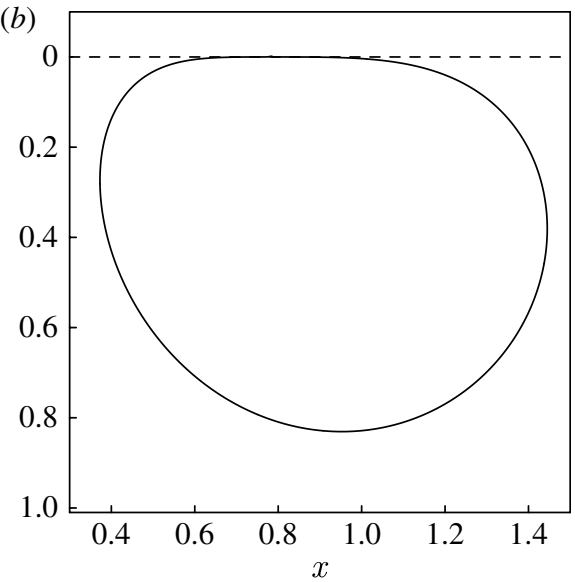

Figure 6. Submerged bodies in $x>0$ that support trapped modes, for the cases $(a) s=0.5$, $h_{0}=0.0145873$ and $(b) s=1.1, h_{0}=0.0013002$.

\section{Asymptotic analysis}

Given that it becomes more difficult to find $h_{0}$ as $s$ gets large, it shall be supposed that there is a critical value of $s$, say $s_{0}$, above which there are no submerged bodies that support trapped modes which are constructed in this way. The existence of this limiting value shall be proved via the use of asymptotic expansions.

Suppose that the dipoles are positioned at $\pm a+\mathrm{i} \varepsilon$, where $|\varepsilon| \ll 1$, so that $z_{0}=a+\mathrm{i} \varepsilon$. Under the assumption that $\varepsilon=h_{0}$, the value of $s$ for which $\psi_{1}=\psi_{2}$ is sought. Put $s=s_{0}-\varepsilon s_{1}=s_{0}-\varepsilon(\alpha+\beta \ln \varepsilon+\gamma \varepsilon+\delta \varepsilon \ln \varepsilon+\cdots)$, where $\alpha, \beta, \gamma$ and $\delta$ are constants to be determined. (The reason for assuming this particular form for $s$ will become apparent.) Finally, put $z=a+\varepsilon u$, so that the stagnation points (in $x>0$ ) are located at as yet unknown locations, say $u=u_{ \pm}$, where $\pm \operatorname{Re}\left(u_{ \pm}\right)>0$ since the stagnation points lie on opposite sides of $x=a$. These forms can be substituted into $w(z)$ given by (3.7), which for convenience is scaled by a factor $(1+s)$, to yield

$$
\begin{aligned}
w(z)= & -\frac{2 z_{0} \mathrm{e}^{-\mathrm{i} a}}{z^{2}-z_{0}^{2}}-\frac{2 \bar{z}_{0} \mathrm{e}^{\mathrm{i} a}}{z^{2}-\bar{z}_{0}^{2}}-2 \mathrm{ie}^{-\mathrm{i} a}(1+s) \int_{0}^{\infty} \frac{\mathrm{e}^{\mathrm{i} m\left(z+z_{0}\right)}}{s m^{3}+m-1-s} \mathrm{~d} m \\
& +2 \mathrm{ie}^{\mathrm{i} a}(1+s) \int_{0}^{\infty} \frac{\mathrm{e}^{\mathrm{i} m\left(z-\bar{z}_{0}\right)}}{s m^{3}+m-1-s} \mathrm{~d} m .
\end{aligned}
$$

We shall write $w(z)=w_{1}(z)+w_{2}(z)$, where $w_{1}(z)$ constitutes the 'dipole terms', given by the first two terms on the right-hand side of (4.1), with the remainder making up the 'integral terms'. The stagnation points are therefore given by

$$
\frac{\mathrm{d} w_{1}}{\mathrm{~d} u}+\frac{\mathrm{d} w_{2}}{\mathrm{~d} u}=0 .
$$

Expanding $w_{1}$ in powers of $\varepsilon$ yields

$$
\begin{aligned}
w_{1}(u)= & \frac{-2(u \cos a+\sin a)}{\varepsilon\left(1+u^{2}\right)}+\frac{\cos a}{a}-\frac{\varepsilon(u \cos a+\sin a)}{2 a^{2}} \\
& +\frac{\varepsilon^{2}\left(\left(u^{2}-1\right) \cos a+2 u \sin a\right)}{4 a^{3}}+O\left(\varepsilon^{3}\right),
\end{aligned}
$$


so that

$$
\frac{\mathrm{d} w_{1}}{\mathrm{~d} u}=\frac{2\left(u^{2}-1\right) \cos a+4 u \sin a}{\varepsilon\left(1+u^{2}\right)^{2}}-\frac{\varepsilon \cos a}{2 a^{2}}+\frac{\varepsilon^{2}(u \cos a+\sin a)}{2 a^{3}}+O\left(\varepsilon^{3}\right) .
$$

The asymptotic derivation of $w_{2}$ and its derivative may be found in appendix A. With these done, the positions of the stagnation points can now be computed up to and including order $\varepsilon^{2}$. To leading order, (4.3) and (A 6) in (4.2) give

$$
u^{2}+2 u \tan a-1=0 \Rightarrow u_{ \pm}=-\tan a \pm \sec a .
$$

It can be seen from (A 1) that the leading order term of $\mathrm{d} w_{2} / \mathrm{d} u$ is $\varepsilon c_{0}\left(s_{0}\right)$, where

$$
c_{0}\left(s_{0}\right)=-2\left(1+s_{0}\right) \mathrm{e}^{-\mathrm{i} a} \int_{0}^{\infty} \frac{m\left(\mathrm{e}^{2 \mathrm{i} a}-\mathrm{e}^{2 \mathrm{i} m a}\right)}{s_{0} m^{3}+m-1-s_{0}} \mathrm{~d} m .
$$

Hence, at the next order, the stagnation points are given by

$$
2\left(u^{2}-1\right) \cos a+4 u \sin a=\left(\frac{\cos a}{2 a^{2}}-c_{0}\left(s_{0}\right)\right) \varepsilon^{2}\left(1+u^{2}\right)^{2} .
$$

Writing $u_{ \pm}=-\tan a \pm \sec a+\varepsilon^{2} q_{ \pm}$, expressions for $q_{+}$and $q_{-}$are found to be, after some manipulation,

$$
q_{ \pm}= \pm \sec ^{2} a\left(\frac{\cos a}{2 a^{2}}-c_{0}\left(s_{0}\right)\right)(\sec a \mp \tan a)^{2} .
$$

It can be seen that $\operatorname{Im}\left(q_{ \pm}\right)$has the same sign as $\mp \operatorname{Im}\left(c_{0}\right)$, and so, if $\operatorname{Im}\left(c_{0}\right) \neq 0$, the stagnation points lie on opposite sides of the free surface, and the body that supports trapped modes ceases to be submerged. It follows that, to retain the possibility of a submerged body,

$$
\operatorname{Im}\left(c_{0}\left(s_{0}\right)\right)=0
$$

For a given value of $a$, this condition can be used to find the critical value $s_{0}$. With $a=\pi / 4, s_{0} \approx 1.276997$ is obtained. This explains the difficulties encountered when trying to obtain numerical results for $a=\pi / 4, s>1.27$.

The next-order terms in the expansion must now be considered to find the depth of the stagnation points below the free surface and also to find a condition on $\varepsilon$ that ensures $\psi_{1}=\psi_{2}$. The stagnation points are determined by

$$
\begin{aligned}
& \frac{2\left(u^{2}-1\right) \cos a+4 u \sin a}{\varepsilon\left(1+u^{2}\right)^{2}}-\frac{\varepsilon \cos a}{2 a^{2}}+\frac{\varepsilon^{2}(u \cos a+\sin a)}{2 a^{3}} \\
& -2 \mathrm{e}^{-\mathrm{i} a} \varepsilon\left(1+s_{0}\right)\left(n_{2}(a)+\mathrm{i} \varepsilon(u+\mathrm{i}) n_{3}(a)\right)-2 \varepsilon^{2}(\alpha+\beta \ln \varepsilon) \mathrm{e}^{-\mathrm{i} a} n_{5}(a) \\
& -2 \mathrm{e}^{-\mathrm{i} a} \varepsilon \frac{1+s_{0}}{s_{0}} \int_{0}^{\infty} \frac{m \mathrm{e}^{\mathrm{i} m \varepsilon(u+\mathrm{i})} e_{a}(m)}{(m+1)^{3}} \mathrm{~d} m=0,
\end{aligned}
$$

where $n_{i}(i=2,3,5)$ and $e_{a}(m)$ are defined in appendix $\mathrm{A}$, and the asymptotic expansion of the integral term is given in (A 7). Put $u_{ \pm}=-\tan a \pm \sec a+$ $\varepsilon^{2} q_{ \pm}+v_{ \pm} \varepsilon^{3} \ln \varepsilon+\varepsilon^{3} r_{ \pm}$, where $v_{ \pm}$and $r_{ \pm}$are to be determined; condition (4.6) determines these in terms of coefficients $\alpha$ and $\beta$ in the expansion of $s$. Then, $u_{ \pm}$ 
can be substituted into the condition $\psi_{1}=\psi_{2}$, that is,

$$
\operatorname{Im}\left(w\left(u_{+}\right)-w\left(u_{-}\right)\right)=0 .
$$

Note that the terms involving $\gamma$ and $\delta$ in (A 4) are constants, and so they cancel in equation (4.7), which will take the form of an equation involving $\varepsilon^{2}$ and $\varepsilon^{2} \ln \varepsilon$ terms. As $\varepsilon$ is arbitrary, the coefficients of these terms must separately be equated to zero, and these two equations can be solved to find $\alpha$ and $\beta$. For the case $a=\pi / 4$, it is found, omitting the details of the calculations, that $\alpha \approx-3.24$ and $\beta \approx-23.2$.

As the stagnation points must lie beneath the free surface, the conditions to be satisfied are, since $\operatorname{Im}\left(q_{ \pm}\right)=0$,

$$
\operatorname{Im}\left(v_{+} \ln \varepsilon+r_{+}\right)>0 \quad \text { and } \quad \operatorname{Im}\left(v_{-} \ln \varepsilon+r_{-}\right)>0 .
$$

Now that $\alpha$ and $\beta$ are known, constraints on $\varepsilon$ can be formulated. It transpires that the necessary conditions (4.8) can be reduced to the inequality $\varepsilon<1.15$, which is clearly valid due to the assumption that $|\varepsilon| \ll 1$. Thus, for a given dipole submergence $\varepsilon$, the approximate value of $s$ that ensures $\psi_{1}=\psi_{2}$ for $a=\pi / 4$ is, up to and including $O(\varepsilon), s=1.276997+\varepsilon(3.24+23.2 \ln \varepsilon)$, provided $\varepsilon$ is small. This last equation agrees well with the numerical results found when $\varepsilon$ is less than approximately $5 \times 10^{-4}$, which corresponds to the range $1.19<s<s_{0}$. For these values of $\varepsilon$, the error in the approximation is at most $0.37 \%$.

\section{Analysis for varying $a$}

Consider $c_{0}\left(s_{0}\right)$, given by (4.4), and recall that it was required to be purely real in order for submerged bodies to exist. The quantity $c_{0}$ shall now be considered to be a function of both $a$ and $s_{0}$.

Given a particular dipole separation $a$, the condition on $c_{0}\left(a, s_{0}\right)$ allows the critical value of the surface tension (above which submerged obstacles cannot be found) to be determined. This is shown in figure 7 , which indicates that a critical surface tension value can be found provided $a$ is not too large. This value increases with the separation of the bodies, and it appears that $s_{0}$ diverges to infinity when $a$ approaches a value close to 0.9 . This case shall now be considered more closely.

First, the path of integration of the integral in (4.4) is rotated (without crossing any singularities) to lie up the imaginary axis. With the substitution $m=\mathrm{i} v$, the integral therefore becomes

$$
c_{0}\left(a, s_{0}\right)=2\left(1+s_{0}\right) \int_{0}^{\infty} \frac{v\left(\mathrm{e}^{\mathrm{i} a}-\mathrm{e}^{-\mathrm{i} a-2 a v}\right)}{-\mathrm{i} s_{0} v^{3}+\mathrm{i} v-1-s_{0}} \mathrm{~d} v .
$$

In the limit $s_{0} \rightarrow \infty$, this becomes

$$
c_{0}\left(a, s_{0}\right)=-2 \int_{0}^{\infty} \frac{v\left(\mathrm{e}^{\mathrm{i} a}-\mathrm{e}^{-\mathrm{i} a-2 a v}\right)}{1+\mathrm{i} v^{3}} \mathrm{~d} v,
$$




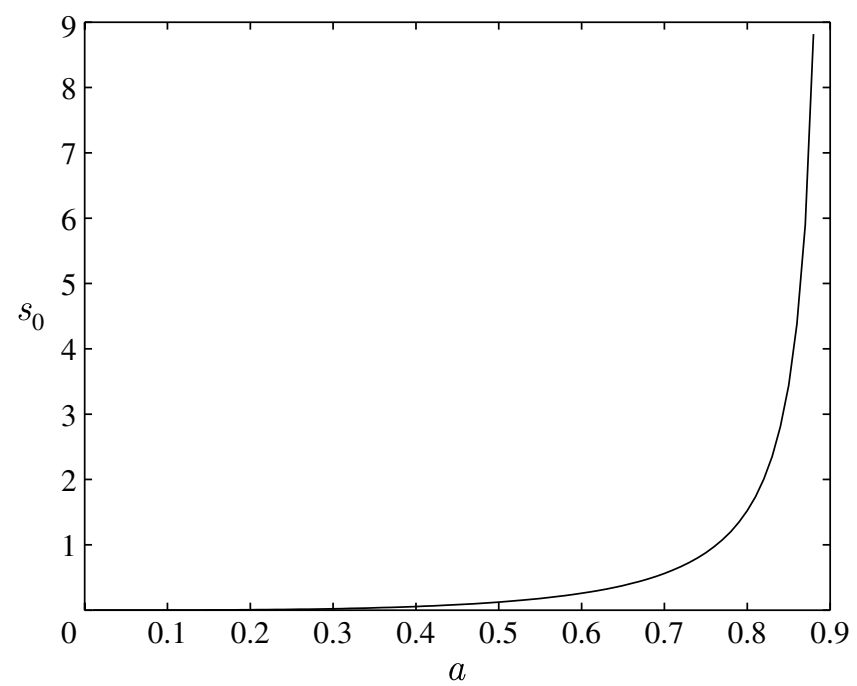

Figure 7. Graph showing how the critical surface tension value varies with $a$.

and so the condition $\operatorname{Im}\left(c_{0}\left(a, s_{0}\right)\right)=0$ yields

$$
\cos a \int_{0}^{\infty} \frac{v^{4}}{1+v^{6}}\left(1-\mathrm{e}^{-2 a v}\right) \mathrm{d} v=\sin a \int_{0}^{\infty} \frac{v}{1+v^{6}}\left(1+\mathrm{e}^{-2 a v}\right) \mathrm{d} v
$$

This is satisfied when $a \approx 0.901924$. Therefore, beyond this value of $a$ there is no critical value for the surface tension, i.e. the submerged bodies which support trapped modes are possible for all values of $s$.

Examining expression (4.4) from a different perspective, it can be supposed that, for every critical value $s_{0}$, there is a minimum dipole separation, say $a_{\text {min }}$, below which there are no submerged obstacles that support trapped modes. Of course, $a_{\text {min }}$ is related to the minimum allowable body separation. For a given $s_{0}, a_{\min }$ is given by figure 7 . It might be expected that there is also a maximum dipole separation $a_{\max }$. Figure 8 shows how the dipole submergence $h_{0}$ varies as a function of $a$, for a few values of $s$. It can be seen that $h_{0} \rightarrow 0$ as $a \rightarrow a_{\min }$, where $a_{\text {min }}$ is considered to be a function of $s$. For the large value $s=100, a_{\text {min }}$ has almost converged to its limiting value as $s \rightarrow$ $\infty$. The figure is strongly suggestive of a constant value for $a_{\max }$, for all $s$ values, that is approximately equal to $\pi / 2$. This indicates why McIver could not construct submerged bodies for values of $a$ between $\pi / 2$ and $\pi$. Other points to note about the graph are that the general trend is of decreasing $h_{0}$ for increasing $s$, although for small $s$ there is an initial increase of $h_{0}$ for values of $a$ centred around 0.9 . In addition, as $s$ increases, the region of validity of $a$ for submerged trapped modes narrows.

Finally, as a matter of interest, numerical experimentation shows that the global maximum value of $h_{0}$ that supports submerged trapped modes is 0.0597177 and is attained when $s=0.013, a=0.7652$. When there is no surface tension present, the maximum obtainable dipole depth is 0.0547949, which occurs when $a=0.7756$. 


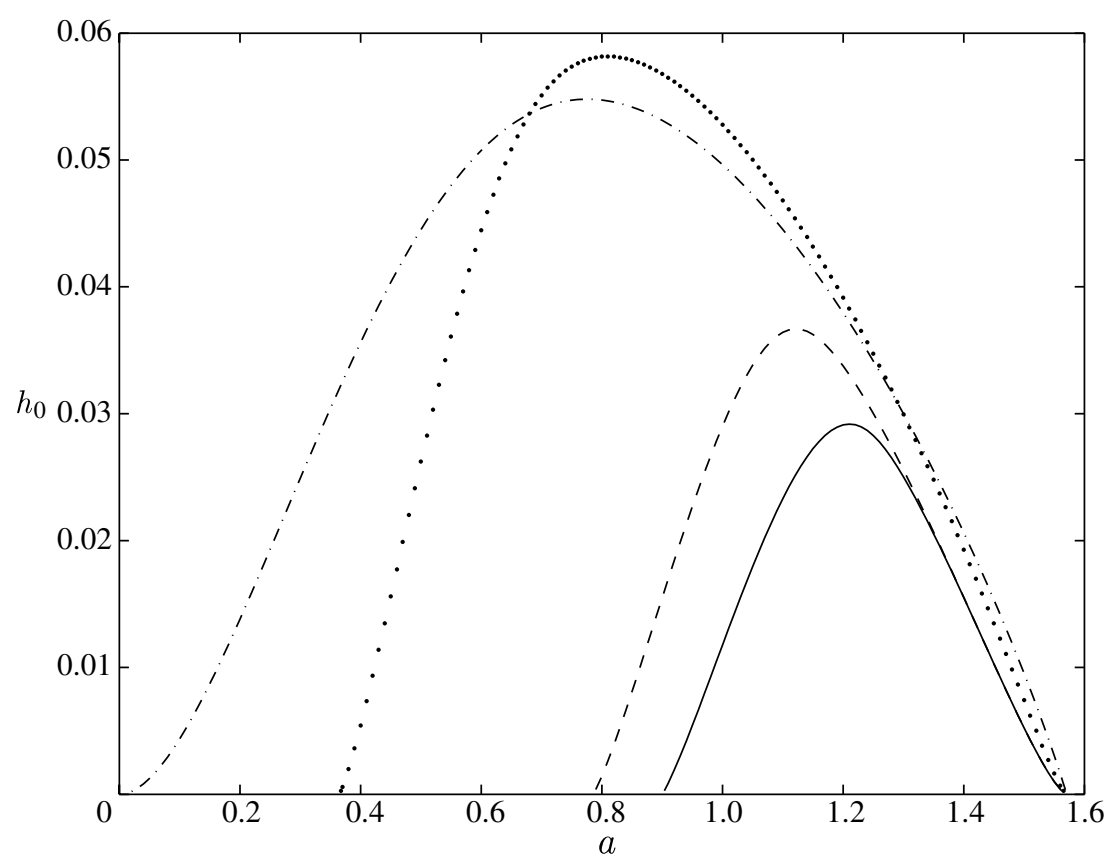

Figure 8. Plot of dipole submergence against dipole separation, for a few values of $s$. The values of $s$ shown are 0 (dash-dotted line), 0.04 (dotted line), 1.277 (dashed line) and 100 (solid line).

\section{Conclusion}

It is shown in this paper that trapped modes supported by immersed rigid bodies can be constructed when surface tension is included in the linearized (inviscid incompressible irrotational) water-wave problem. Moreover, its exclusion from the problem is not always justifiable, as its inclusion in a particular submerged body example changes the qualitative (i.e. topological) nature of the streamline pattern. This is not just a hypothetical result - the breakdown of the existence of localized solutions about these submerged bodies occurs at physically realistic wavelengths, provided that the parameter $a$ is chosen appropriately. For example, when $a=0.1, s_{0} \approx 0.00055$, which in water corresponds to a wavelength of $0.74 \mathrm{~m}$.

The breakdown of the result may have been foreseeable, as the structures originally produced in McIver (2000) are particularly unstable, in the sense that there is only one pair of streamlines that can be interpreted as the boundaries of bodies. This is in contrast to the numerous choices for the streamlines that were available in McIver (1996), where the inclusion of surface tension makes no difference to the final outcome. Nevertheless, it might be advisable to consider surface tension in any future works, as its inclusion could bring about unexpected results. For example, current investigations are ongoing as to its effect on wave trapping on the submerged bodies discussed by Porter $(2002 a, b)$.

One interesting line of further work concerns the extension of known uniqueness proofs to include surface tension. Of particular interest are those proofs that rely on certain geometric criteria being met, such as those given by 
Simon \& Ursell (1984). The introduction of capillarity may alter these conditions, possibly extending or reducing cases of uniqueness; this is currently being investigated by the authors.

\section{Appendix A}

The integral part of (4.1) is written as

$$
\begin{aligned}
w_{2}(u) & =-2 \mathrm{ie}^{-\mathrm{i} a}(1+s) f_{0}^{\infty} \frac{\mathrm{e}^{\mathrm{i} m(2 a+\varepsilon(u+\mathrm{i}))}}{d(m)} \mathrm{d} m+2 \mathrm{ie}^{\mathrm{i} a}(1+s) f_{0}^{\infty} \frac{\mathrm{e}^{\mathrm{i} m \varepsilon(u+\mathrm{i})}}{d(m)} \mathrm{d} m \\
& =2 \mathrm{ie}^{-\mathrm{i} a}(1+s) \int_{0}^{\infty} \frac{\mathrm{e}^{\mathrm{i} m \varepsilon(u+\mathrm{i})} e_{a}(m)}{d(m)} \mathrm{d} m,
\end{aligned}
$$

where $e_{a}(m)=\mathrm{e}^{2 \mathrm{i} a}-\mathrm{e}^{2 \mathrm{i} m a}$ and $d(m)=s m^{3}+m-1-s$; we shall also write $d_{0}(m)=$ $s_{0} m^{3}+m-1-s_{0}$, so that $s=s_{0}-\varepsilon s_{1}$ yields $d(m)=d_{0}(m)-\varepsilon s_{1}\left(m^{3}-1\right)$. Thus

$$
-\frac{\mathrm{i}}{2} \mathrm{e}^{\mathrm{i} a} w_{2}=\left(1+s_{0}-\varepsilon s_{1}\right) \int_{0}^{\infty} \frac{\mathrm{e}^{\mathrm{i} m \varepsilon(u+\mathrm{i})} e_{a}(m)}{d_{0}(m)-\varepsilon s_{1}\left(m^{3}-1\right)} \mathrm{d} m,
$$

which after some work yields the expansion

$$
\begin{aligned}
-\frac{\mathrm{i}}{2} \mathrm{e}^{\mathrm{i} a} w_{2}= & \left(1+s_{0}\right) \int_{0}^{\infty} \frac{\mathrm{e}^{\mathrm{i} m \varepsilon(u+\mathrm{i})} e_{a}(m)}{d_{0}(m)} \mathrm{d} m \\
& +\varepsilon s_{1} \int_{0}^{\infty} \frac{m\left(m^{2}-1\right) \mathrm{e}^{\mathrm{i} m \varepsilon(u+\mathrm{i})} e_{a}(m)}{d_{0}^{2}(m)} \mathrm{d} m \\
& +\varepsilon^{2} s_{1}^{2} \int_{0}^{\infty} \frac{m\left(m^{2}-1\right)\left(m^{3}-1\right) \mathrm{e}^{\mathrm{i} m \varepsilon(u+\mathrm{i})} e_{a}(m)}{d_{0}^{3}(m)} \mathrm{d} m+\cdots .
\end{aligned}
$$

The last two integrals in this expression can be expanded to give all terms up to and including $O\left(\varepsilon^{2}\right)$, using the standard series expansion of $\mathrm{e}^{x}$. However, it can be seen that, when the exponential in the first integrand is expanded in this way up to $O\left(\varepsilon^{2}\right)$, the integral fails to converge (which is why $\ln \varepsilon$ terms are needed in the asymptotic expansion of $s$ ). This problem can be dealt with by splitting the integral as

$$
\begin{aligned}
\int_{0}^{\infty} \frac{\mathrm{e}^{\mathrm{i} m \varepsilon(u+\mathrm{i})} e_{a}(m)}{d_{0}(m)} \mathrm{d} m= & \int_{0}^{\infty} \mathrm{e}^{\mathrm{i} m \varepsilon(u+\mathrm{i})} e_{a}(m)\left(\frac{1}{d_{0}(m)}-\frac{1}{s_{0}(m+1)^{3}}\right) \mathrm{d} m \\
& +\int_{0}^{\infty} \frac{\mathrm{e}^{\mathrm{i} m \varepsilon(u+\mathrm{i})} e_{a}(m)}{s_{0}(m+1)^{3}} \mathrm{~d} m .
\end{aligned}
$$

Again, the expansion of $\mathrm{e}^{x}$ allows the first integral on the right to be expanded (up to $O\left(\varepsilon^{2}\right)$ ), and the second may be evaluated to the required order using the following relation

$$
2 \int_{0}^{\infty} \frac{\mathrm{e}^{-m z}}{(m+1)^{3}} \mathrm{~d} m=1-z+z^{2} \mathrm{e}^{z} E_{1}(z)=f(z), \quad \text { say, }
$$


valid for $\operatorname{Re}(z) \geq 0$, with

$$
f(z) \sim 1-z-z^{2}(\ln (z)+C) \quad(z \rightarrow 0),
$$

where $C$ denotes the Euler-Mascheroni constant. Recalling from $\S 4$ that $s_{1}=\alpha+$ $\beta \ln \varepsilon+\gamma \varepsilon+\delta \varepsilon \ln \varepsilon+\cdots$ leads to an expression for $w_{2}$ of the form

$$
\begin{aligned}
-\frac{\mathrm{i}}{2} \mathrm{e}^{\mathrm{i} a} w_{2} \sim & \left(1+s_{0}\right)\left[n_{1}+\mathrm{i} \varepsilon(u+\mathrm{i}) n_{2}-\frac{1}{2} \varepsilon^{2}(u+\mathrm{i})^{2} n_{3}\right] \\
& +\frac{1+s_{0}}{s_{0}} \int_{0}^{\infty} \frac{\mathrm{e}^{\mathrm{i} m \varepsilon(u+\mathrm{i})} e_{a}(m)}{(m+1)^{3}} \mathrm{~d} m+\varepsilon(\alpha+\beta \ln \varepsilon+\gamma \varepsilon+\delta \varepsilon \ln \varepsilon) n_{4} \\
& +\mathrm{i} \varepsilon^{2}(\alpha+\beta \ln \varepsilon)(u+\mathrm{i}) n_{5}+\varepsilon^{2}(\alpha+\beta \ln \varepsilon)^{2} n_{6},
\end{aligned}
$$

where $n_{i}(i=1, \ldots, 6)$, constants which depend on the spacing $a$, are given by

$$
\begin{aligned}
n_{i} & =\int_{0}^{\infty} m^{i-1} e_{a}(m)\left(\frac{1}{d_{0}(m)}-\frac{1}{s_{0}(m+1)^{3}}\right) \mathrm{d} m, \quad i=1,2,3, \\
n_{i} & =\int_{0}^{\infty} \frac{m^{i-3}\left(m^{2}-1\right) e_{a}(m)}{d_{0}^{2}(m)} \mathrm{d} m, \quad i=4,5 \\
n_{6} & =\int_{0}^{\infty} \frac{m\left(m^{2}-1\right)\left(m^{3}-1\right) e_{a}(m)}{d_{0}^{3}(m)} \mathrm{d} m .
\end{aligned}
$$

The integral remaining in (A 4) is evaluated up to the correct order using (A 2) and (A 3) to give

$$
\begin{aligned}
& 2 \int_{0}^{\infty} \frac{\mathrm{e}^{\mathrm{i} m \varepsilon(u+\mathrm{i})} e_{a}(m)}{(m+1)^{3}} \mathrm{~d} m=\mathrm{e}^{2 \mathrm{i} a} f(\varepsilon-\mathrm{i} \varepsilon u)-f(\varepsilon-2 \mathrm{i} a-\mathrm{i} \varepsilon u) \\
& \sim \mathrm{e}^{2 \mathrm{i} a}\left[1-\varepsilon(1-\mathrm{i} u)-\varepsilon^{2}(1-\mathrm{i} u)^{2}[\ln \varepsilon+\ln (1-\mathrm{i} u)+\gamma]\right] \\
& \quad-\left[f(-2 \mathrm{i} a)+\varepsilon(1-\mathrm{i} u) f^{\prime}(-2 \mathrm{i} a)+\frac{1}{2} \varepsilon^{2}(1-\mathrm{i} u)^{2} f^{\prime \prime}(-2 \mathrm{i} a)\right] .
\end{aligned}
$$

The derivative of the complex potential $w_{2}$ can now be written down as

$$
\begin{aligned}
-\frac{\mathrm{i}}{2} \mathrm{e}^{\mathrm{i} a} \frac{\mathrm{d} w_{2}}{\mathrm{~d} u}= & \mathrm{i}\left(1+s_{0}\right)\left[\varepsilon n_{2}-\varepsilon^{2}(1-\mathrm{i} u) n_{3}\right] \\
& +\mathrm{i} \varepsilon \frac{1+s_{0}}{s_{0}} \int_{0}^{\infty} \frac{m \mathrm{e}^{\mathrm{i} m \varepsilon(u+\mathrm{i})} e_{a}(m)}{(m+1)^{3}} \mathrm{~d} m+\mathrm{i} \varepsilon^{2}(\alpha+\beta \ln \varepsilon) n_{5}+O\left(\varepsilon^{3}\right),
\end{aligned}
$$

whose integral term may be determined up to $O\left(\varepsilon^{2}\right)$, via differentiation of (A 5), as

$$
\begin{aligned}
& \frac{\mathrm{i} \varepsilon}{2} \frac{\left(1+s_{0}\right)}{s_{0}}\left[\mathrm{e}^{2 \mathrm{i} a}+f^{\prime}(-2 \mathrm{i} a)\right. \\
& \left.\quad+2 \varepsilon \mathrm{e}^{2 \mathrm{i} a}(1-\mathrm{i} u)[\ln \varepsilon+\ln (1-\mathrm{i} u)+1 / 2+\gamma]+\varepsilon(1-\mathrm{i} u) f^{\prime \prime}(-2 \mathrm{i} a)\right] .
\end{aligned}
$$




\section{References}

Billingham, J. \& King, A. C. 2000 Wave motion. Cambridge, UK: Cambridge University Press.

Evans, D. V. 1968 The influence of surface tension on the reflection of water waves by a plane vertical barrier. Proc. Camb. Philos. Soc. 64, 795-810.

Evans, D. V. \& Porter, R. 1998 An example of non-uniqueness in the two-dimensional linear waterwave problem involving a submerged body. Proc. R. Soc. A 454, 3145-3165. (doi:10.1098/rspa. 1998.0295)

Harter, R., Simon, M. J. \& Abrahams, I. D. In preparation. The effect of surface tension on localized free surface oscillations about surface-piercing bodies.

Hocking, L. M. 1987 Waves produced by a vertically oscillating plate. J. Fluid Mech. 179, 267-281. (doi:10.1017/S0022112087001526)

Lighthill, J. 1978 Waves in fluids. Cambridge, UK: Cambridge University Press.

McIver, M. 1996 An example of non-uniqueness in the two-dimensional linear water wave problem. J. Fluid Mech. 315, 257-266. (doi:10.1017/S0022112096002418)

McIver, M. 2000 Trapped modes supported by submerged obstacles. Proc. R. Soc. A 456, 1851-1860. (doi:10.1098/rspa.2000.0589)

McIver, P. \& McIver, M. 1997 Trapped modes in an axisymmetric water-wave problem. Q. J. Mech. Appl. Math. 50, 165-178. (doi:10.1093/qjmam/50.2.165)

McIver, P. \& Newman, J. N. 2003 Trapping structures in the three-dimensional water-wave problem. J. Fluid Mech. 484, 283-301. (doi:10.1017/S0022112003004397)

McIver, M. \& Porter, R. 2002 Trapping of waves by a submerged elliptical torus. J. Fluid Mech. 456, 277-293. (doi:10.1017/S0022112001007327)

Porter, R. $2002 a$ Surface wave scattering by submerged cylinders of arbitrary cross-section. Proc. R. Soc. A 458, 581-606. (doi:10.1098/rspa.2001.0885)

Porter, R. $2002 b$ Trapping of water waves by pairs of submerged cylinders. Proc. R. Soc. A 458, 607-624. (doi:10.1098/rspa.2001.0871)

Rhodes-Robinson, P. F. 1991 The effect of surface tension on the progressive waves due to incomplete vertical wave-makers in water of infinite depth. Proc. R. Soc. A 435, 293-319. (doi:10.1098/rspa.1991.0146)

Simon, M. J. \& Ursell, F. 1984 Uniqueness in linearized two-dimensional water-wave problems. J. Fluid Mech. 148, 137-154. (doi:10.1017/S0022112084002287)

Thorne, R. C. 1953 Multipole expansions in the theory of surface waves. Proc. Camb. Philos. Soc. 49, 707-716.

Ursell, F. 1950 Surface waves on deep water in the presence of a submerged circular cylinder. Proc. Camb. Philos. Soc. 46, 141-158. 